\title{
Wavelet based Scheme to Improve Performance of Hearing under Noisy Environment
}

\author{
Jayant J. Chopade \\ Research Scholar, \\ Matoshri College of Engineering \& Research \\ Center \\ Nashik, SPPU, Pune
}

\author{
N.P. Futane, PhD \\ Government of College of Engineering \\ Awasari, Pune, India \\ SPPU, Pune
}

\begin{abstract}
Conventionally the testing of hearing aid algorithm is accomplished by conducting listening test on hearing impaired, but these tests are not only time consuming but also causes exhaustion, especially in aged patients. Simulation based testing proves to be better option for preliminary evaluation of developed algorithm. A novel methodology based on wavelet transform is designed for dichotic presentation. Among different wavelet families, daubechies \& symlet are chosen due to their pre-eminence among others. The performance of developed algorithm has been tested on four normal hearing subjects under noisy environment with SNR of $3 \mathrm{db}, 0 \mathrm{db},-3 \mathrm{db} \&-6 \mathrm{db}$ in prerecorded phonetically balanced words. Comparative result analysis of performance measures like perception rate and perception time shows the outperformance of processed over unprocessed signals.
\end{abstract}

\section{General Terms}

Hearing impaired, Wavelet transform, SNR

\section{Keywords}

Dichotic presentation, Sensorineural, Binaural, Spectral masking, Speech Processing.

\section{INTRODUCTION}

In Sensorineural hearing impaired people the auditory filters are broader than normal in increased spectral masking. Masking takes place mainly at the outer level of ear and splitting of speech into two complementary signals and presenting them dichotically for two ears can help in minimizing the effect of increased masking in persons with sensorineural hearing impairment [1].

The wavelet transform is used in signal processing, due to the ability of wavelet transforms to present a time-frequency (or time-scale) representation of signals as the wavelet transform uses a variable-width window (wide at low frequencies and narrow at high frequencies).

Wavelet analysis is considered as a bank of band pass filters. The wavelet filter bank allows a better representation of both the temporal and the place pitch in the speech signals [2], have designed WP filter bank and incorporated into a commercial ACE (Advanced Combinational Encoder) strategy for speech processing in cochlear implants Averaged results of speech intelligibility tests have shown that the mixed WP filter-bank leads to significantly better speech perception performance than the fast Fourier transform as used in the commercial ACE strategy. Yao, J. et [3] investigated the application of an enhanced signal processing scheme called bionic wavelet transform (BWT). Authors concluded that application of the BWT in cochlear implants has a number of advantages, including improved recognition rates for vowels and consonants, reduction in the number of channels in cochlear implant, reduction in the average stimulation duration of words, better noise tolerance and higher speech intelligibility rates. Abhjit Karmarkal et al.[4] have proposed a criterion to select the optimal wavelet packet based on the Zwicker's model critical band structure [5]. Authors obtained optimal WP tree for different sampling frequencies and results were compared with other $\mathrm{CB}$ motivated WP trees. M.T.Kolte et al. [6] Proposed modified wavelet packets algorithm using symlet family. Author's claimed that recognition scores for processed scheme of wavelet packets were improved by $3.33 \%$ to $22.23 \%$. and the response time were reduced. P.A.Dhulekar et al.[7] have proposed improved wavelet packets. The proposed method was evaluated using VCV speech material. Author concluded that results obtained by proposed method were better than comb filter results. In present work modified wavelet packet algorithm of Daubechies, Symlet wavelets families were developed. In which eight bands are created based on auditory filters of quasi octave bandwidth. Four alternate bands are combined for even-odd dichotic presentation. The inverse wavelet packet transform was used to synthesize speech components from the wavelet packet representation. To synthesize the speech component, wavelet coefficients were used. Listening tests on four normal hearing people using phonetically balanced (PB) words have been conducted.

The paper is organized into four sections. Section I introduces the need of the proposed system and also reviews the different techniques proposed by the different researchers to overcome various problems related to the hearing impaired using wavelet transform. Section II discusses the design of modified wavelet packet. Section III includes listening tests. Section IV shows experimental results and discussion. Section V concludes the paper.

\section{DESIGN OF MODIFIED WAVELET PACKET}

The processing schemes are developed as spectral splitting with mixed wavelets packets based on eight frequency bands as the performance by hearing-impaired saturated around eight channels, while performance by normal-hearing subjects sustained to 12-16 channels in higher background noise [8]. The number of channels desired to obtain high levels of speech understanding is still the subject of discussion [9]. Two different algorithms were developed based on modified wavelet packet with daubechies, and symlet wavelet functions. Daubechies and symlets are orthogonal wavelets that have the highest number of vanishing moments for a givens support width. The inverse wavelet packet transform was used to synthesize speech components from the wavelet packet representation. To synthesize the speech component, wavelet coefficients are used. 
In modified wavelet packet, discrete wavelet transform is applied at first level of decomposition and wavelet packet for further three levels to obtain eight bands having quasi octave bandwidth. For each level daubechies, symlet family with same order of decomposition was used. Following figure 1 shows modified wavelet packet tree.

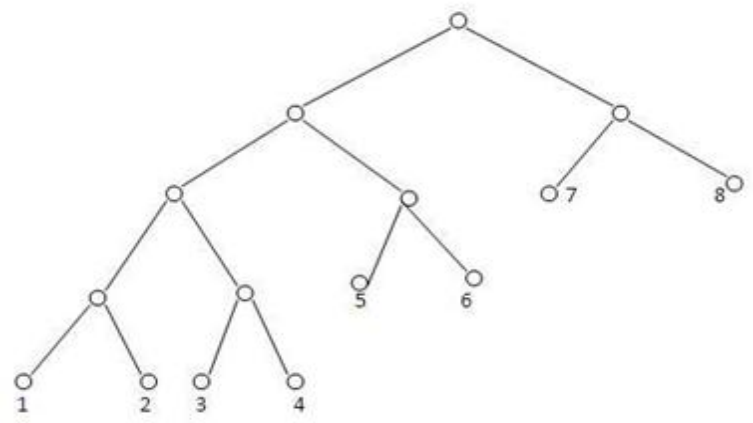

Figure 1: Modified wavelet packet tree

The mother functions used are based on the daubechies i.e. $\mathrm{dB} 4$, symlet i.e. sym9 families.

Table 1 shows the eight bands in alternate fashion for evenodd index with center and passband frequency for each band in $\mathrm{KHz}$.

Table 1 Pass band frequencies

\begin{tabular}{|c|c|c|c|c|c|}
\hline \multicolumn{3}{|c|}{ Filter for left ear } & \multicolumn{3}{c|}{ Filter for right ear } \\
\hline Band & $\begin{array}{c}\text { Centre } \\
\text { frequency } \\
\mathrm{KHz}\end{array}$ & $\begin{array}{c}\text { Passband } \\
\text { frequency } \\
\mathrm{KHz}\end{array}$ & Band & $\begin{array}{c}\text { Centre } \\
\text { frequency } \\
\mathrm{KHz}\end{array}$ & $\begin{array}{c}\text { Passband } \\
\text { frequency } \\
\mathrm{KHz}\end{array}$ \\
\hline 1 & 0.15625 & $0-0.3125$ & 2 & 0.46875 & $0.312-0.625$ \\
\hline 3 & 0.78125 & $0.625-0.937$ & 4 & 1.0937 & $0.9375-1.25$ \\
\hline 5 & 1.5625 & $1.25-1.875$ & 6 & 2.1875 & $1.875-2.5$ \\
\hline 7 & 3.125 & $2.5-3.75$ & 8 & 4.375 & $3.75-5$ \\
\hline
\end{tabular}

The stepwise workflow of the new approach of modified wavelet packet is presented in following algorithm:

\subsection{Pseudo-Algorithm}

1) Read audio input signal $x(n)$ (with noise) of length N.

2) Perform wavelet packet decomposition of $x(n)$ up to level 5

3) Construct the modified wavelet packet tree Tmod by rejoining following nodes of the original tree $\mathrm{T}$ :

[1, 3, 5 and 7] and [2, 4, 6 and8].

The modified tree will have only 8 nodes.

4) Selectively reconstruct the modified wavelet tree to get two output signals: one for left ear and other for right ear, as follows. a. In modified tree, make all 4 approximate coefficients nodes [1, 3, 5 and 7] zero while keeping detail coefficients nodes as it is and reconstructed that tree.

b. In modified tree, make all 4 detail coefficients nodes [2, 4, 6 and 8] zero keeping approximate coefficients nodes as it is and reconstructed that tree.

\section{THE LISTENING TESTS}

The listening tests were conducted to evaluate speech processing schemes to find the most suitable one for improving speech perception for persons having sensorineural type of hearing loss. The speech materials used in tests were phonetically balanced monosyllabic words.

Acoustically isolated room was used to conduct the listening test. The words were presented to the two ears through headphones and all the words had approximately same intensity of sound given to each ear. The testing was done on four normal hearing peoples, with simulated sensorineural impairment. The sensorineural impairment was simulated with masker added at SNR values of $3 \mathrm{~dB}, 0 \mathrm{~dB} .-3 \mathrm{~dB} \&-6 \mathrm{~dB}$.

Listening tests were conducted on four normal hearing subjects. Four subjects belong to different age group.

\section{LISTENING TEST RESULTS AND DISCUSSION}

Listening tests are carried out to measure two performance parameters that are perception rate and perception time. Comparative analysis of these parameters for processed and unprocessed signal was evaluated. The detail analysis of these result are shown in following subsections.

\subsection{Perception Rate}

The perception rate of four subjects under the three category; unprocessed signal (US), processed signal by daubechies 4 (PS-dB4) and processed signal by symlet (PS-sym9) has been shown in Table 2.

The perception rate has been measured under the presence of noise with SNR level of $3 \mathrm{~dB}, 0 \mathrm{~dB},-3 \mathrm{~dB}$ and $-6 \mathrm{~dB}$.

Table 2: Perception Rate (\%) of US, PS(db4) and PS(sym9) for 4 subjects

\begin{tabular}{|l|c|c|c|c|}
\hline \multicolumn{1}{|c|}{ Subjects } & $\mathbf{3 ~ d B}$ & $\mathbf{0 ~ d B}$ & $\mathbf{- 3 ~ d B}$ & $\mathbf{- 6 ~ d B}$ \\
\hline S1 (US) & & & & \\
\hline S1 (PS-db4) & 95 & 100 & 90 & 80 \\
\hline S1 (PS-sym9) & 90 & 100 & 100 & 90 \\
\hline S2 (US) & 70 & 95 & 70 & 60 \\
\hline S2 (PS-db4) & 80 & 95 & 75 & 70 \\
\hline S2 (PS-sym9) & 80 & 100 & 80 & 75 \\
\hline S3 (US) & 100 & 90 & 95 & 75 \\
\hline S3 (PS-db4) & 100 & 90 & 93.33 & 75 \\
\hline S3 (PS-sym9) & 95 & 100 & 100 & 75 \\
\hline S4 (US) & 93.33 & 100 & 90 & 90 \\
\hline S4 (PS-db4) & 100 & 100 & 95 & 96.67 \\
\hline S4 (PS-sym9) & 95 & 100 & 100 & 96.67 \\
\hline
\end{tabular}




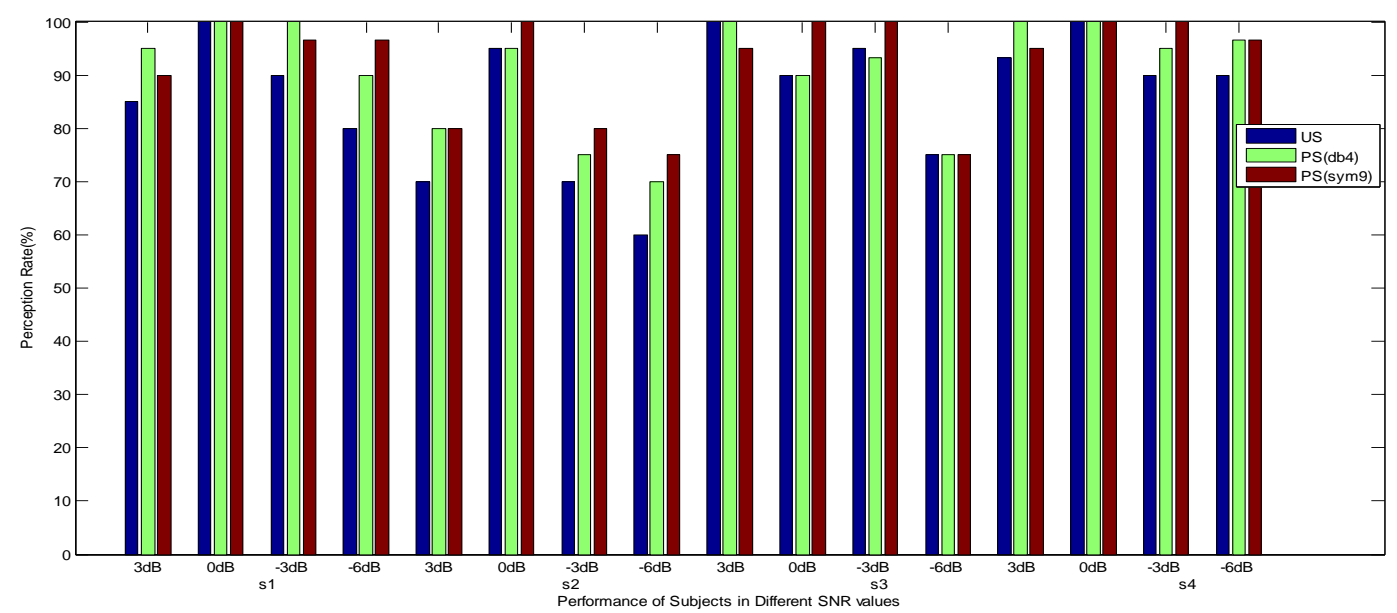

Figure 2 Performance of subject with different SNR values vs Perception rate

Table 3: Relative Improvement in Perception Rate (\%)

\begin{tabular}{|l|c|c|c|c|}
\hline Subjects & $\mathbf{3 ~ d B}$ & $\mathbf{0 ~ d B}$ & $\mathbf{- 3} \mathbf{d B}$ & $\mathbf{- 6} \mathbf{~ d B}$ \\
\hline $\mathrm{S} 1(\mathrm{db} 4)$ & 10 & 0 & 10 & 10 \\
\hline $\mathrm{S} 1$ (sym9) & 5 & 0 & 6.67 & 6.67 \\
\hline $\mathrm{S} 2(\mathrm{db} 4)$ & 10 & 0 & 5 & 10 \\
\hline $\mathrm{S} 2(\mathrm{sym} 9)$ & 10 & 5 & 10 & 15 \\
\hline $\mathrm{S} 3(\mathrm{db} 4)$ & 0 & 0 & -1.67 & 0 \\
\hline $\mathrm{S} 3(\mathrm{sym} 9)$ & -5 & 10 & 5 & 0 \\
\hline $\mathrm{S} 4(\mathrm{db} 4)$ & 6.67 & 0 & 5 & 6.67 \\
\hline $\mathrm{S} 4(\mathrm{sym} 9)$ & 1.67 & 0 & 10 & 6.67 \\
\hline
\end{tabular}

Figure 2 shows the comparison of processed signal obtained using daubechies and symlet families with unprocessed signal. Bar chart shows the increased perception rate for S1, S2 and S4 subject by 5 to $10 \%$.

The relative improvement in perception rate has been shown in Table3 while comparative analysis for relative improvement in perception rate has been shown in figure 3 . The results show the desired relative improvement in three subject s1, s2 and s4. For the SNR of 3dB, the subject s3 has shown better improvement compared to SNR of $0 \mathrm{~dB},-3 \mathrm{~dB}$ and $-6 \mathrm{~dB}$. At $0 \mathrm{~dB}$ the relative improvement is better for symlet compared to daubechies wavelet family.

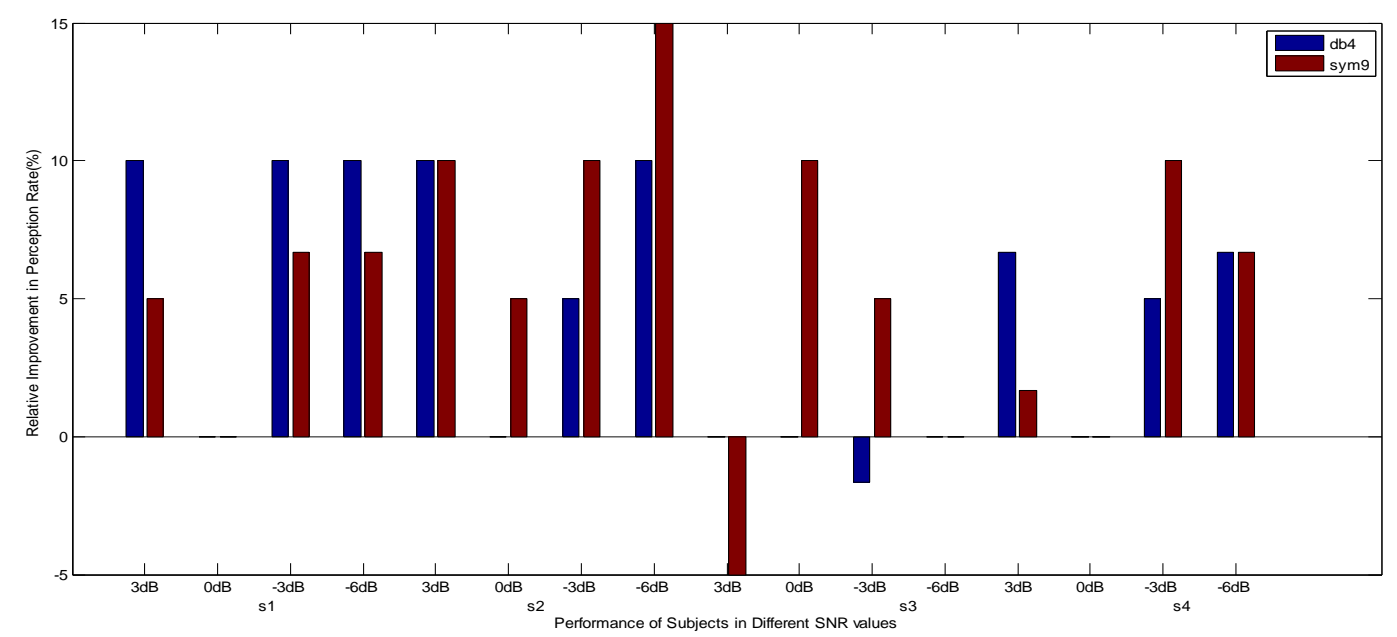

Figure 3 Relative Improvement in Perception rate (\%)

\subsection{Perception Time}

Perception Time is the time interval between speech material presented dichotically to subjects and the response given by subjects. The perception time of four subjects under the three category; unprocessed signal (US), processed signal by daubechies 4 (PS-dB4) and processed signal by symlet (PSsym9) has been shown in Table 4.The perception time has been measured under the presence of noise with SNR level of $3 \mathrm{~dB}, 0 \mathrm{~dB},-3 \mathrm{~dB}$ and $-6 \mathrm{~dB}$. 
Table 4: Perception Time of US, PS(db4) and PS(sym9)

\begin{tabular}{|l|l|l|l|l|}
\hline \multicolumn{1}{|c|}{ Subjects } & $\mathbf{3 ~ d B}$ & $\mathbf{0 ~ d B}$ & $\mathbf{- 3} \mathbf{d B}$ & $\mathbf{- 6 ~ d B}$ \\
\hline S1 (US) & & & & \\
\hline S1 (PS-db4) & 2.65 & 2.65 & 4.19 & 4.11 \\
\hline S1 (PS-sym9) & 2.55 & 2.59 & 3.01 & 4.09 \\
\hline S2 (US) & 3.08 & 4.11 & 4.21 & 4.23 \\
\hline S2 (PS-db4) & 2.90 & 2.91 & 4.07 & 3.75 \\
\hline S2 (PS-sym9) & 2.49 & 2.88 & 3.09 & 3.32 \\
\hline S3 (US) & 2.74 & 2.85 & 2.90 & 2.79 \\
\hline S3 (PS-db4) & 2.74 & 2.85 & 2.99 & 2.79 \\
\hline S3 (PS-sym9) & 2.99 & 2.71 & 2.70 & 2.69 \\
\hline S4 (US) & 2.26 & 2.23 & 3.05 & 2.45 \\
\hline S4 (PS-db4) & 2.10 & 2.20 & 2.91 & 2.32 \\
\hline S4 (PS-sym9) & 2.05 & 2.20 & 2.00 & 2.11 \\
\hline
\end{tabular}

Figure 4 shows the comparison of processed signal obtained using daubechies and symlet families with unprocessed signal. Bar chart shows the reduced perception time for $\mathrm{S} 1, \mathrm{~S} 2$ and S4 subject by 0.02 to $1.23 \mathrm{sec}$.

Table 5: Relative Improvement in Perception Time (sec)

\begin{tabular}{|l|c|c|c|c|}
\hline Subjects & $\mathbf{3 ~ d B}$ & $\mathbf{0 ~ d B}$ & $\mathbf{- 3 ~ d B}$ & $\mathbf{- 6 ~ d B}$ \\
\hline S1 (db4) & 0.04 & 0.06 & 1.17 & 0.02 \\
\hline S1 (sym9) & 0.10 & 0.10 & 0.40 & 0.54 \\
\hline S2 (db4) & 0.18 & 1.20 & 0.14 & 0.48 \\
\hline S2 (sym9) & 0.59 & 1.23 & 1.12 & 0.91 \\
\hline S3 (db4) & 0.00 & 0.00 & -0.09 & 0.00 \\
\hline S3 (sym9) & -0.25 & 0.14 & 0.20 & 0.10 \\
\hline S4 (db4) & 0.16 & 0.03 & 0.14 & 0.13 \\
\hline S4 (sym9) & 0.21 & 0.03 & 1.05 & 0.34 \\
\hline
\end{tabular}

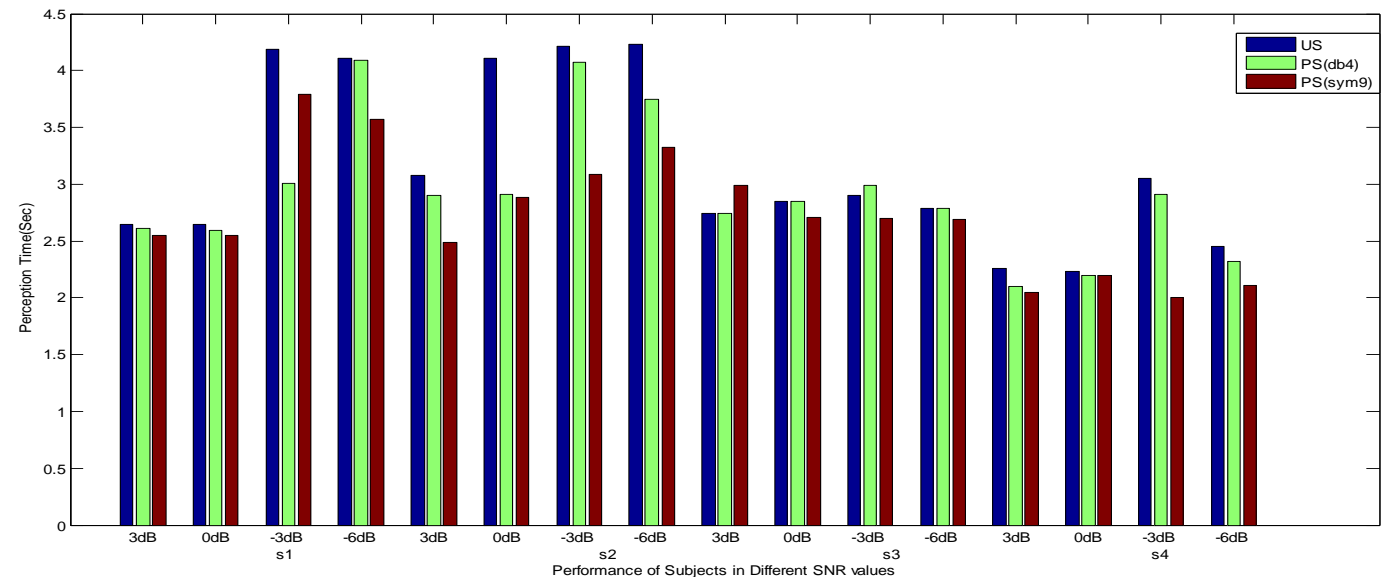

Figure 4 Performance of subject with different SNR values vs Perception time

The relative improvement in perception time has been shown in Table 5 while comparative analysis for relative improvement in perception time has been shown in figure 5 . The results show the desired relative improvement in three subject s1, s2 and s4. For the SNR of 3dB, all the subject except $\mathrm{s} 3$ has shown better improvement compared to SNR of $0 \mathrm{~dB},-3 \mathrm{~dB}$ and $-6 \mathrm{~dB}$. At $0 \mathrm{~dB}$ the relative improvement is almost zero for all the subjects except processed signal obtained by symlet wavelet families.

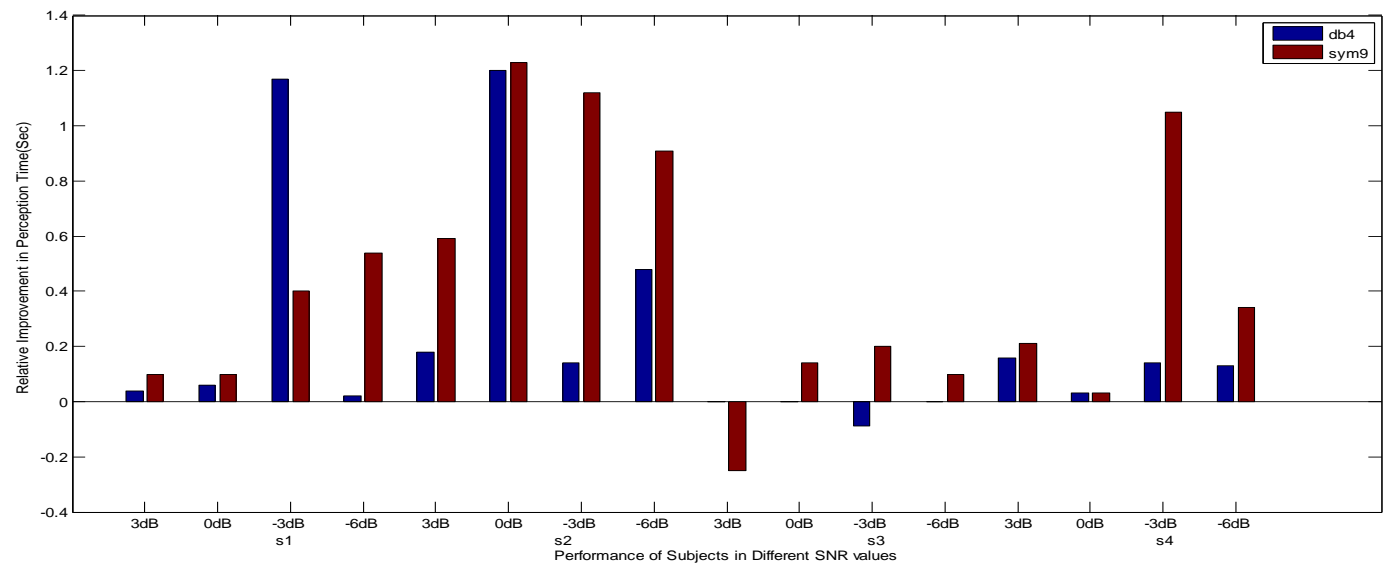

Figure 5 Relative Improvement in Perception time (sec) 


\section{CONCLUSIONS}

The proposed method proves to be desired alternative to test the applicability of developed algorithm for hearing aid without involvement of actual hearing impaired. In this work speech signal were spitted into two complementary signal using wavelet transform with different families and order. Resulting split signal components having probability to mask each other were presented to left and right ears which reduce the effect of increased auditory masking.

The results from the listening tests shows that the perception rate have been increased in the range of 5 to $10 \%$ while perception time has been reduced in the range of 0.02 to 1.23 sec. The observed results show the outperformance of processed signals over unprocessed.

Further investigation may also be carried out for assessing the effect of binaural dichotic presentation on the perception of more speech words and also on sentences.

\section{REFERENCES}

[1] B. C. J. Moore. An Introduction to Psychology of Hearing. London: Academic, 4th ed. 1997, pp. 89-140

[2] Nogueira, W., Giese, A., Edler, B., and Buchner, A. (2006). "Wavelet Packet Filterbank for Speech Processing Strategies in Cochlear Implants," Proc. IEEE Int. Conf. Acoustic Speech, Signal Processing (ICASSP '06). vol. 5, 14-19.

[3] Yao, J., and Zhang, Y. (2002). "The Application of Bionic Wavelet Transform to Speech Signal Processing in Cochlear Implants using Neural Network Simulations," IEEE Transactions on Biomedical Engineering, vol. 49(11), 1299-1309.

[4] Abhijit Karmarkal ,Arun Kumar and R.K.Patney, "Design of Optimal Wavelet Packet Trees Based on Auditory Perception Criterion", IEEE SIGNAL PROCESSING LETTERS, VOL. 14, NO. 4, APRIL 2007.

[5] E. W. Zwicker, "Subdivision of audible frequency rangeinto critical bands (Freqenzgruppen)," J. Acoust . Soc. Am.,vol. 33, pp. 248, 1961.

[6] Mahesh T.Kolte, D.S.Chaudhari "Evaluation of speech processing schemes to improve perception of sensorinural hearing impaired"in Current Science, Vol.98,No 5, 2010.

[7] P.A.Dhulekar and Dr.S.L.Nalbalwar." Optimization of Wavelet Packets to Minimize the Effect of Spectral
Masking for Improving Speech Perception", International Journal of Computer Applications (0975 8887) Volume 25- No.11, July 2011.

[8] Baskent and Deniz. "Speech recognition in normal hearing and sensorineural hearing loss as a function of the number of spectral channels," J. Acoust. Soc. Am., 2006, vol. 120(5), pp. 2908-2925.

[9] Loizou, P. C., (1999). "Signal processing techniques for cochlear implants" IEEE Magazine in Medicine and Biology Engg. vol. 18(3), 34-46.

[10] L. R. Rabiner and R. W. Schafer. Digital Processing of the Speech Signals, Englewood Cliffs, NJ: Prentice Hall, 1978, pp.35-53.

[11] D. S. Chaudhari and P. C. Pandey. "Dichotic presentation of speech signal using critical filter bank for bilateral sensorineural hearing impaired". Proceedings of 16th International Conf. on Acoustics, Seattle, Washington, 1998, vol. 1, pp. 213-214.

[12] CHABA. "Speech-perception aids for hearing-impaired people: Current status and needed research," J. Acoust. Soc. Am. vol. 90, 637-683, 1991.

[13] P.N.Kulkarni and P.C. Pandey. "Optimizing the comb filters for spectral splitting of speech to reduce the effect of spectral masking," in Proc. International conference on signal processing, Communication and Networking, Chennai, India, Jan 2008 pp.69-73.

[14] Lunner, T., and Hellgren, J. (1991). "A digital filterbank hearing aid- design, implementation, and evaluation," Proc. in IEEE ASSP, vol. 5, 3661-3664.

[15] J. R. Dubno and A. B. Schaefer. "Comparison of frequency selectivity and consonant recognition among hearing-impaired and masked normal-hearing listeners," J. Acoust. Soc. Am., 1992, vol. 91(4), pp. 2110-2121.

[16] Rao, R. M., and Bopardikar, A. S. (2001). Wavelet Transform Introduction to Theory and Applications (Addison Wesley Longmman Pte. Ltd., Delhi)

[17] Daubechies, I. (1992). Ten Lectures on Wavelets, Philadelphia: SIAM, CBMS- NSF Regional Conference in Applied Mathematics 61.

[18] Valerie Hanson, Kofi Odame, "Real-Time Embedded Implementation of the Binary Mask Algorithm for Hearing Prosthetics", IEEE Transactions on Biomedical Circuits and Systems, Vol. 8, No.4, August 2014. 